\section{The race is on}

Efforts to combat the spread of drug-resistant malaria in Africa received a blow this month from research in Science $(305,1124 ; 2004)$ that examines the origins of resistance, but hope emerged from another study in Nature $(430,900-904 ; 2004)$ that introduces a synthetic version of an antimalarial drug.

Sulfadoxine pyrimethamine, commonly known as Fansidar, has become the drug of choice for malaria in Africa. Yet the specter of widespread pyrimethamine resistance is foreshadowed by events in Asia, where the drug is often useless. One assumption is that resistance is arising de novo in parasite strains on the African continent. Cally Roper et al. dispel this notion, reporting that some of the more resistant strains in Africa have been imported from southeast Asia. The researchers examined microsatellite DNA flanking mutations in the gene that encodes dihydrofolate reductase, which underlies resistance to pyrimethamine. Alleles with three mutations, which confer high treatment failure rates, originated from southeast Asia. Why just one origin? The researchers speculate that complex compensatory mutations are required to restore parasite fitness.

While public health measures to prevent migration of resistant strains might help stem the tide, so would better drugs. The new compound, patterned after the plant-derived drug artemisinin, emerges from a joint drug development effort from the Medicines for Malaria Venture. Artemisinin is recommended by the World Health Organization as a second agent in malaria drug combinations-but the drug is relatively expensive owing to the extraction process, has side effects and requires a days-long drug regimen. Jonathan Vennerstrom et al. report that in mice the synthetic drug is more potent and acts for longer than the plant-derived drug. The compound has now entered clinical trials.

\section{DNA drop-off}

Trypanosoma cruzei is a weird parasite. One might think that this agent of Chagas disease, in an effort to maximally co-opt its host, would have jettisoned every extraneous bit of protein, DNA and lipid. But it carries a cumbersome load-the largest amount of extranuclear DNA of any eukaryote. In the 23 July issue of Cell (118, 175-186; 2004), Nadjar Nitz et al. show that the parasite drops off some of this excess baggage in its vertebrate host, leaving behind bits of integrated mitochondrial DNA.

The mitochondrial DNA of this organism is not only large; it is fussy. The DNA is organized into 'mini' circles encoding RNA that mediates RNA editing of mitochondrial RNA encoded on 'maxi' circles. An analysis of 13 people with advanced Chagas disease, as well as infected chickens and rabbits showed that the mini-circle DNA integrates into the host genome. What's more, the DNA also seems to be transmitted through the germline and passed on to offspring, as the researchers showed in chickens.

T. cruzei can cause heart disease and nerve damage years after the initial infection. Whether DNA integration contributes to chronic disease, is part of some evolutionary scheme to modify the host genome, or is simply a peculiar molecular accident is an open question.

Written by Charlotte Schubert

\section{Remain calm and alert}

Most agents that increase wakefulness, such as cocaine, also increase anxiety. In the August 19 issue of Neuron (43, 487-497; 2004), Yan-Ling $\mathrm{Xu}$ et al. find another exception, neuropeptide S (NPS), pulled from a screen for ligands for orphan G-protein receptors. Mice with NPS injected into the brain slept less than their untreated counterparts and moved around more. They also were less anxious, as measured by four different tests - for instance, burying fewer marbles in a marble-strewn cage. Arousal is likely to be modulated by multiple neuronal systems, and NPS is expressed in a previously undefined cluster of cells near a region implicated in wakefulness, the locus coeruleus. If harnessed in a drug, the NPS system holds promise for the treatment disorders such as depression, which is often accompanied by excessive anxiety and sleep.

\section{Making sense of sensitivity}

The dopamine precursor L-dopa can ease symptoms Parkinson disease, in which dopaminergic transmission goes awry. But the drug has drawbacks in the long-term, often leading to dyskinesiaexcessive, uncontrolled movement. In the August 4 issue of Journal of Neuroscience (24, 7007-7014; 2004), Jean-Christophe Corvol et al. examine the basis for this debilitating side effect.

Previous work had shown that dyskinesia seems to result from D1 dopamine hypersensitivity. But levels of the D1 receptor remain constant with treatment, suggesting that downstream effectors account for the exaggerated response to dopamine. Corvol et al. homed in one such effector: the G-protein subunit $\mathrm{G} \alpha_{\text {olf }}$. Studies in post-portem tissue of Parkinson patients and in rats suggested that L-dopa treatment results in elevated levels of $\mathrm{G} \alpha_{\text {olf }} \cdot \mathrm{G} \alpha_{\text {olf }}$ links the D1 receptor to adenylyl cyclase, and increased $\mathrm{G} \alpha_{\text {olf }}$ expression was found to increase cyclase activity. Thus enhanced signaling through $\mathrm{G} \alpha_{\text {olf }}$ may contribute to dyskinesia.

\section{Chromosome control}

Aneuploidy often arises in human cancers as cells lose control of the orderly progression of cell division and chromosome separation. One common notion is that selection works directly on cancer cells to produce aberrations in chromosome number. A study in the 12 August issue of Nature $(430,797-802 ; 2004)$ challenges this viewpoint, showing that aneuploidy can arise through a more prosaic process-inactivation of the cell cycle regulator retinoblastoma.

Many studies suggest that aneuploidy arises from defects in the spindle checkpoint that regulates mitotic progression. Some of the checkpoint regulators, such as Mad2, sense improper microtubule tension or attachment to microtubules. Yet loss-of-function mutations in genes for such regulators are rarely observed in human tumors. Eva Hernando et al. found that inactivation of retinoblastoma results not only in dysregulated cell cycle progression but also in high Mad2 expression. What's more, Mad2 overexpression led to aneuploidy in cell culture experiments and correlated with aneuploidy and poor prognosis in human tumors. The critical molecular intermediary between retinoblastoma and Mad2 is the retinoblastoma target E2F, which transcriptionally regulates Mad2.

Erratum: The research highlight "Touching T cells" in the August 1 issue $(10,785 ; 2004)$ contained errors. The name of the first author was incorrect; it is Ramnik Xavier. The citation is to The Journal of Cell Biology $(166,173-178 ; 2004)$, and the description of the figure should read, "DIg1 in green; actin, red; APC, blue." 